Check for updates

Cite this: Chem. Commun., 2020, 56, 13661

Received 24th August 2020 Accepted 7th October 2020

DOI: $10.1039 / \mathrm{d} 0 \mathrm{cc} 05681 \mathrm{~b}$

rsc.li/chemcomm

\section{Reactivity prediction in aza-Michael additions without transition state calculations: the Ames test for mutagenicity $\dagger$}

\author{
Piers A. Townsend (iD ${ }^{a b}$ and Matthew N. Grayson (iD *b
}

\begin{abstract}
Animal testing remains a contentious ethical issue in predictive toxicology. Thus, a fast, versatile, low-cost quantum chemical model is presented for predicting the risk of Ames mutagenicity in a series of 1,4 Michael acceptor type compounds. This framework eliminates the need for transition state calculations, and uses an intermediate structure to probe the reactivity of aza-Michael acceptors. This model can be used in a variety of settings e.g., the design of targeted covalent inhibitors and polyketide biosyntheses.
\end{abstract}

With increasing scientific, ethical, and societal drivers to reduce animal testing, the development of novel predictive in silico approaches for chemical safety assessment is vital. In silico methods are low-cost, and are amongst the fastest, most dynamic approaches for the initial safety assessment of chemicals. ${ }^{1}$ For these reasons, there is great interest in adopting computational approaches in toxicological regulation and legislation. ${ }^{2}$ Cancer, or carcinogenicity, continues to be a toxicological endpoint of great concern, with over 9 million people dying every year from cancer related illness. ${ }^{3}$ Mutagenicity is a well-studied endpoint to predict carcinogenicity and is a process by which inheritable cancer-causing genetic changes are induced. ${ }^{4}$ When DNA mutations arise, the first step often involves the formation of a covalent adduct between a DNA nucleobase and an electrophilic exogenous chemical agent. ${ }^{4,5}$ The Ames test is a widely used in vitro bioassay for identifying carcinogens and has mutagenicity as the observed endpoint. ${ }^{6}$ In drug discovery, a positive Ames test result on novel drug candidates can be a significant roadblock in their development; it is indicative of a hazardous chemotype and can often halt clinical trials on potential therapeutics. ${ }^{7}$ Thus, the in silico prediction of Ames test results provides a number of advantages; a rapid, low-cost assessment at the earliest possible stage in development will save time and money, along with circumnavigating the need for animal testing. Transition

\footnotetext{
${ }^{a}$ Centre for Sustainable Chemical Technologies, University of Bath, Claverton Down, Bath, BA2 7AY, UK

${ }^{b}$ Department of Chemistry, University of Bath, Claverton Down, Bath, BA2 7AY, UK. E-mail:M.N.Grayson@bath.ac.uk

$\dagger$ Electronic supplementary information (ESI) available. See DOI: 10.1039/d0cc05681b
}

state modeling (TSM) has been used extensively for studying chemical reaction mechanisms. ${ }^{8,9}$ The chemical reactivity of 1,4 Michael Acceptors (MAs) in aza-Michael additions underpins many chemical processes and this reactivity can be assessed with TSM approaches. ${ }^{10}$ Examples include DNA adduct formation and the design of novel candidates for targeted covalent inhibitors (TCIs). ${ }^{11}$ TCIs are rationally designed to first bind with their target protein through intermolecular interactions, followed by an important covalent bond forming reaction between the TCI and nucleophilic moieties e.g., amine side chains. The covalent modification leads to increased binding affinities and thus, increased potency. Further, aza-Michael additions are common in ribosomal and non-ribosomal peptide biosynthesis, and have recently been adopted as a strategy in macrolactam polyketide biosynthesis. ${ }^{12}$

In 2019, we published a model that utilised DFT TSM to predict the Ames test results of 30 1,4 Michael acceptors, with activation free energy and LUMO energies showing significant correlation with mutagenic risk. ${ }^{13}$ Our results showed that a higher level of theory in the molecular geometry optimisation procedure had a direct effect on the quality of the reactivity predictions made on the original dataset of 19 MAs. ${ }^{14}$ Despite significant predictive potential shown by the model, TSM can be costly, exhaustively time consuming and challenging for the non-expert. Thus, examination of the toxicant-target potential energy surface without the use of TSM is beneficial for fast, low cost predictions in applications such as industrial chemical risk assessment and the design of novel TCIs. In the reaction between 1,4 MAs and biological nucleophiles, a high energy intermediate (HEI) enolate exists along the reaction pathway (see Fig. 1) ${ }^{15}$ Calculations on these high energy intermediate structures, that closely resemble the transition states in line with Hammond's postulate, may be broadly extended to the wide variety of toxicologically relevant covalent phenomena discussed above. ${ }^{16}$ This study presents a novel framework for the prediction of Ames test results using the HEI formed in the reaction between 1,4 Michael acceptors and nucleophilic moieties such as DNA nucleobases. Further, we directly compare 

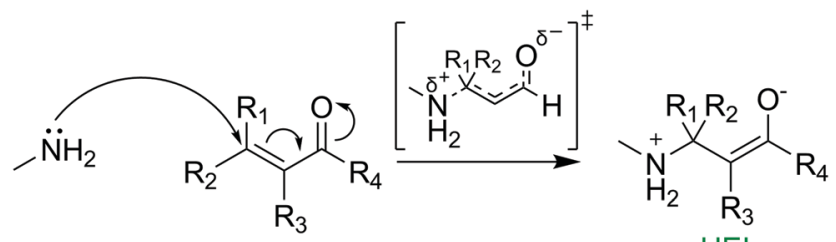

Fig. 1 Mechanism for the formation of an enolate intermediate in the reaction between a typical 1,4 Michael acceptor and methylamine.

the use of fast, low-cost semi-empirical calculations with slower yet more accurate DFT calculations.

In this study, 28 1,4 Michael acceptors were selected from our previously published dataset (see ESI, $\dagger$ Fig. S1). Two structures were eliminated from the original dataset. Firstly, 2-bromoacrylic acid was removed due to it being the only carboxylic acid in the group. Secondly, 3-(dichloromethylidene)pyrrolidine-2,5-dione was removed due to an inconclusive Ames test result, leading to uncertainty in the correct classification. ${ }^{17}$ For the 28 compounds, experimental Ames test results towards the TA100 strain of S. typhimurium (without S9) were taken from two resources: the OECD QSAR Toolbox and a dataset published in 2010 by Cordeiro and co-workers. ${ }^{18,19}$ Of the 28 compounds, 14 were previously classified as Ames positive, whilst a further 14 were classified as Ames negative. To ensure faster calculation times and reduced computational costs, methylamine was chosen as a surrogate for the most reactive DNA nucleobase, guanine (see Fig. 1 and Fig. S2, ESI $\dagger$ ). ${ }^{20}$ Conformational searches were performed using the MMFF force field, on both the reactants and HEIs of the chosen compounds using Schrödinger's Macromodel (Version 11.3). ${ }^{21}$ Successfully converged conformations were further optimised in Gaussian 16 (Rev. A.03) using both semiempirical and DFT methods (see ESI $\dagger$ for full details). ${ }^{22}$ All semiempirical optimizations used the Austin Model 1 (AM1) level of theory followed by single point energy calculations at the B3LYPD3(BJ)/def2-TZVPP-IEFPCM(water) level of theory. DFT structures were calculated at the B3LYP-D3(BJ)/def2-TZVPP-IEFPCM(water) level of theory. These methods were chosen due to extensive use in previous studies of organic chemical reaction mechanisms. ${ }^{23-25}$

Our previously published model utilised activation energies and molecular orbital energies to predict the Ames test result of 1,4 Michael acceptors. ${ }^{13}$ Despite the significant predictive potential shown by the model, TSs can be time-consuming and challenging to locate computationally. Further, conformational flexibility can lead to hundreds of individual TSs, ensuring high computational costs. The HEI structure is a minimum on the potential energy surface, as opposed to a saddle point, and thus involves a much simpler calculation procedure than that required to locate TSs (see Fig. S3, ESI $\dagger$ ). This demands the question: can the energy difference between the HEI and the reactants $\left(\Delta G_{\mathrm{AM} 1} / \Delta G_{\mathrm{DFT}}\right)$ be used as a quantum chemical descriptor for Ames test predictions (see Fig. S4, ESI $\dagger$ )? Throughout this work, energies of the reactants and high energy intermediates were calculated. $\Delta G_{\mathrm{AM} 1 / \mathrm{DFT}}$ is computed as follows:

$$
\Delta G_{\mathrm{AM} 1 / \mathrm{DFT}}=G_{\mathrm{HEI}}-\left(G_{\mathrm{MA}}+G_{\mathrm{N}}\right)
$$

Table 1 Tabulated thermochemistry obtained for the $\mathbf{2 8}$ compounds in this study. $\Delta G_{A M 1}$ and $\Delta G_{D F T}$ are in $\mathrm{kcal} \mathrm{mol}^{-1}$. All $\Delta G_{A M 1}$ values were calculated at the B3LYP-D3(BJ)/def2tzvpp-IEFPCM-(water)//AM1 level of theory. The Ames positive region is shown in red, Ames negative region in green, and the area of uncertainty (AOC) can be seen in yellow

\begin{tabular}{|c|c|c|c|c|}
\hline Compound & CAS-No & $\Delta \mathrm{G}_{\mathrm{AM} 1}$ & $\Delta \mathrm{G}_{\mathrm{DFT}}$ & Ames Result \\
\hline & 1 15743-13-2 & 24.0 & -17.0 & Positive \\
\hline & 7 165606-93-9 & 18.0 & -15.3 & Positive \\
\hline 13 & 3 3787-28-8 & 17.8 & -13.6 & Positive \\
\hline & 3 2648-51-3 & N/A & -11.1 & Positive \\
\hline 11 & 1 1193-54-0 & 30.1 & -10.8 & Positive \\
\hline 14 & 4 61203-01-8 & 37.4 & 4.7 & Positive \\
\hline 18 & 8 14925-39-4 & 25.5 & 9.2 & Positive \\
\hline & $688552-47-0$ & 27.3 & 9.7 & Positive \\
\hline & 4 107-02-8 & 26.7 & 11.0 & Positive \\
\hline & 5 78-94-4 & 27.8 & 12.1 & Positive \\
\hline 15 & 5 101328-96-5 & 30.0 & 12.6 & Positive \\
\hline 12 & $27364-09-2$ & 13.8 & 14.0 & Positive \\
\hline 19 & $94170-30-3$ & 28.9 & 14.1 & Positive \\
\hline & $107-86-8$ & 27.5 & 15.5 & Inconclusive \\
\hline 26 & $6141-32-2$ & 33.0 & 20.1 & Negative \\
\hline 20 & 0 922-63-4 & 31.8 & 20.2 & Positive \\
\hline 28 & 8 4513-36-4 & 32.7 & 20.3 & Negative \\
\hline & $779-06-1$ & 33.1 & 22.5 & Negative \\
\hline 24 & $497-86-9$ & 38.3 & 23.8 & Negative \\
\hline 27 & 7 2397-76-4 & 38.2 & 24.0 & Negative \\
\hline & 2 92694-51-4 & 42.0 & 24.1 & Negative \\
\hline & $814-78-8$ & 34.0 & 24.5 & Negative \\
\hline 23 & $645-62-5$ & 35.4 & 24.6 & Negative \\
\hline 25 & $2873-97-4$ & 35.0 & 24.6 & Negative \\
\hline 22 & 5234-68-4 & 38.6 & 26.9 & Negative \\
\hline & 3 13747-73-4 & 29.3 & 28.0 & Negative \\
\hline & $78-59-1$ & N/A & 30.3 & Negative \\
\hline 10 & $80-71-7$ & 53.1 & 30.5 & Negative \\
\hline
\end{tabular}

where $G_{\mathrm{HEI}}$ is the quasi-harmonic free energy of the high energy intermediate, $G_{\mathrm{MA}}$ is the quasi-harmonic free energy of the 1,4 MA, and $G_{\mathrm{N}}$ is the quasi-harmonic free energy of methylamine.

Austin Model 1 Semi-empirical calculations have an accuracy known to lie between molecular mechanics (MM) and QM approaches such as DFT and Hartree-Fock. ${ }^{26}$ Despite the decrease in accuracy compared to higher level methods, semi-empirical calculations are much faster than DFT methods, and thus, show desirable characteristics for the prediction of toxicological phenomena, such as Ames mutagenicity. ${ }^{27}$ In total, the HEI for 26 of the $\mathbf{2 8}$ compounds were successfully optimised using $\mathrm{AM} 1$, and the HEI energy difference $\left(\Delta G_{\mathrm{AM} 1}\right)$ showed wideranging values from 13.8 to $53.1 \mathrm{kcal} \mathrm{mol}^{-1}$ (see Table 1). Ames positive compounds ranged from 13.8 to $37.4 \mathrm{kcal} \mathrm{mol}^{-1}$ whilst Ames negative compounds ranged from 29.3 to $53.1 \mathrm{kcal} \mathrm{mol}^{-1}$. The average $\Delta G_{\mathrm{AM} 1}$ for Ames positive compounds was $26.1 \mathrm{kcal} \mathrm{mol}^{-1}$ whilst a higher average of $36.2 \mathrm{kcal} \mathrm{mol}^{-1}$ was seen in Ames negative compounds. It is clear that the average energy difference of $10.1 \mathrm{kcal}$ $\mathrm{mol}^{-1}$ between Ames positive and Ames negative compounds is significant. Of the 26 compounds calculated, only two false positives were apparent; two Ames negative compounds ( $\mathbf{1}$ and $\mathbf{8}$, see below for further details) sat within the expected $\Delta G_{\mathrm{AM} 1}$ region for Ames positive compounds (see Table 1). A single Ames positive compound (14) fell within the expected $\Delta G_{\mathrm{AM} 1}$ range for Ames negative compounds. Thus, only a single false negative prediction was observed with the AM1 method. Upon inspecting the optimized structures of compounds 3 and 9, it was apparent that a stable HEI could not be isolated at the AM1 level of theory. The optimization results consistently resulted in nucleophile-electrophile distances greater 
than $5 \AA$ A. A number of attempts were made to obtain these intermediates, including geometry optimization with constraints at the $\mathrm{C}-\mathrm{N}$ atomic coordinates. Once the constrained structure had been optimised to a minimum, the constraint was removed, and a further optimization was performed. The resulting intermediates could not be readily found on the AM1 potential energy surface. Despite these interesting results, for a highly inexpensive computational approach with calculation times much shorter than DFT, often sitting below one minute for AM1 optimizations, a clear trend of separation in $\Delta G_{\mathrm{AM} 1}$ exists at the semi-empirical AM1 level of theory following DFT SPE correction. Many commonly used (Q)SAR chemical descriptors show greater variation in their values when included in published models. ${ }^{28,29}$ Thus, the HEI energy difference $\Delta G_{\mathrm{AM} 1}$ shows great promise for use in multivariate (Q)SAR models targeted towards 1,4 MA toxicity prediction.

Density Functional Theory (DFT) Compared to semi-empirical methods such as AM1, DFT is known to be slower yet more accurate for energies and molecular geometries. ${ }^{30}$ For 23 of 28 compounds in the dataset, HEIs were successfully found and optimised at the B3LYP-D3(BJ)/def2tzvpp level of theory within the IEFPCM model (water). Compared to AM1, a narrower range of 4.7-30.5 kcal mol ${ }^{-1}$ was found for the HEI energy difference $\Delta G_{\mathrm{DFT}}$. The Ames positive compounds showed $\Delta G_{\mathrm{DFT}}$ values ranging from 4.7-20.2 kcal mol ${ }^{-1}$, whilst Ames negative compounds ranged from $20.1-30.5 \mathrm{kcal} \mathrm{mol}^{-1}$. The average $\Delta G_{\mathrm{DFT}}$ for Ames positive and Ames negative compounds was 12.0 and $24.1 \mathrm{kcal} \mathrm{mol}{ }^{-1}$ respectively, leading to an average energy difference of $12.1 \mathrm{kcal} \mathrm{mol}^{-1}$ between mutagenic and nonmutagenic compounds. For five compounds $(3,11,13,17,21)$, the HEI could not be found using DFT. However, significant results were observed for each of these structures. There were two similarities between each of the five failed compounds: (i) all compounds have experimentally determined Ames positive results and (ii) each compound had at least one chlorine atom at the $\beta$-carbon. For each of the five compounds, the optimised structures were all verified minima on the potential energy surface. However, as opposed to the HEI, a product-type state was observed for each compound, along with the elimination of hydrogen chloride (see Fig. S5, ESI $\dagger$ ). The free energy of these product states can be termed $G_{\mathrm{PS}}$. When $\Delta G_{\mathrm{DFT}}$ was computed for the five failed compounds, $G_{\mathrm{HEI}}$ was replaced with $G_{\mathrm{PS}}$ and as a result, negative values for $\Delta G_{\mathrm{DFT}}$ were obtained (see Table 1). These results can be explained by the role that chlorine plays as a leaving group; weak bases are known to be effective leaving groups. ${ }^{31}$ Further, there is evidence that chlorine containing compounds are often positively classified in the Ames test. The published dataset from Kazius and Bursi highlights a number of chlorine containing toxicophores and their associated mutagenic risk: 15 of $18 \alpha$-chlorothioalkanes, 22 of 26 chloroalkenes, 10 of
10 polyhaloalkenes, 18 of 19 1-chloroethyl and 29 of $32 \beta$-haloethoxys were experimentally classified as Ames positive. ${ }^{32}$ The product states observed in the chlorine containing 1,4 MAs are indicative of stable adduct formation upon exposure to methylamine and thus, nitrogen containing nucleobases such as guanine. From a practical perspective, it is worth noting that no further effort was required to obtain $G_{\mathrm{PS}}$ and it is simply the outcome of geometry optimisation and subsequent data analysis. The HEI energy difference calculated for compound 1 led to interest in its mutagenic risk. From our dataset, compound $\mathbf{1}$ was the only experimentally classified Ames negative compound showing

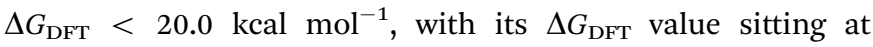
$15.5 \mathrm{kcal} \mathrm{mol}^{-1}$. This result led to re-examination of the toxicological literature for this structure. The OECD have previously reported "a failure to product coherent results" for compound 1. ${ }^{33}$ Although Ames negative results have previously been reported for compound $\mathbf{1}$, mutagenic activity and Ames positive results have also been reported, leading to uncertainty in the mutagenic risk for this chemical. These results likely explain the magnitude of $\Delta G_{\mathrm{DFT}}$ for compound 1 ; by placing $\Delta G_{\mathrm{DFT}}$ into the Ames positive region, this method successfully captures the mutagenic potential shown by this compound in its OECD assessment. Further, although its $\Delta G_{\mathrm{DFT}}$ lies in the Ames positive region, it has the highest $\Delta G_{\mathrm{DFT}}$ value of all structures in the positive region, thereby placing it closer to the negative region than other positive compounds. This may account for the uncertainty in its experimentally derived Ames test result. The energies of $\Delta G_{\mathrm{DFT}}$ obtained show excellent ability to categorise the Ames test result in 1,4 MAs. There is an area of uncertainty (AOU) at $20 \mathrm{kcal} \mathrm{mol}^{-1}$, with one Ames positive compound (20) at $20.2 \mathrm{kcal} \mathrm{mol}^{-1}$ and a one Ames negative compound (26) at $20.1 \mathrm{kcal} \mathrm{mol}^{-1}$. Benigni et al, however, previously showed that compound 20 exhibits a relatively low molar mutagenicity when compared to other values in their dataset. $^{34}$ The model constructed in this work shows a divide between $\Delta G_{\mathrm{DFT}}$ values calculated for Ames positive (mutagenic) and Ames negative (non-mutagenic) compounds. The AOU at $20 \mathrm{kcal} \mathrm{mol}^{-1}$ does exist, and thus, compounds in this region should be treated with caution. Structural features of AP and AN compounds are highlighted and discussed (see ESI, $\dagger$ Fig. S7). To improve the usability of this model, and to utilise this method for the prediction of reactivity in 1,4 MAs, we have proposed a formal protocol (see Fig. 2 and ESI $\dagger$ ). To ensure our model was consistent across methods, we performed three sets of additional calculations on the AOU in which either the DFT functional, basis set or solvent model was changed. The results of these calculations showed that $\Delta G_{\mathrm{DFT}}$ can be used to predict Ames test results at multiple levels of theory (see ESI, $\dagger$ Fig. S8). Further, to test our DFT model, calculations were performed on

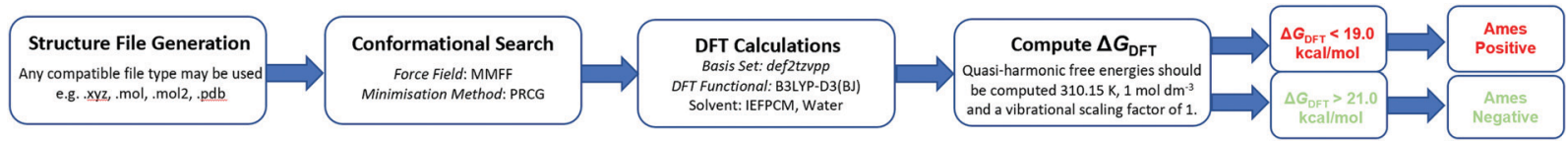

Fig. 2 Flow diagram demonstrating the suggested workflow for predicting the Ames test result of a 1,4 MA. 
two common Michael acceptor type pharmaceuticals: warfarin and embelin (minor truncation, see ESI $\dagger$ ). $\Delta G_{\text {DFT }}$ values of 34.3 and $29.6 \mathrm{kcal} \mathrm{mol}^{-1}$ were respectively obtained, agreeing with experiment, and placing them in the Ames negative region. ${ }^{35}$

This work has presented a rapid, low-cost procedure for assessing the mutagenic risk of 1,4 Michael acceptor type compounds. In the reaction between 1,4 MAs and methylamine, a high energy intermediate is present on the reaction pathway following the $\mathrm{C}-\mathrm{N}$ bond forming TS. The use of intermediate structures in place of transition state structures allows for faster, low-cost data acquisition. From a practical standpoint, intermediate structures (minima) are much simpler to compute than TSs (saddle points). The high energy intermediate energy differences $\left(\Delta G_{\mathrm{AM} 1} / \Delta G_{\mathrm{DFT}}\right)$ were calculated and compared at two levels of theory: the semi-empirical method AM1 and DFT. It was shown that AM1 provides a suitable, low-cost framework for categorising Ames test results. However, false negative predictions did occur, and a few compounds could not be optimised at the AM1 level of theory. DFT results showed excellent performance in categorising Ames test results based on the calculated value of $\Delta G_{\mathrm{DFT}}$. It can be deduced that compounds with $\Delta G_{\mathrm{DFT}}<19.0 \mathrm{kcal} \mathrm{mol}^{-1}$ are likely to be Ames positive, whilst compounds with $\Delta G_{\mathrm{DFT}}>21.0 \mathrm{kcal} \mathrm{mol}^{-1}$ are likely to be Ames negative. This method can be used in the preliminary safety assessment of new and pre-existing pharmaceutically relevant 1,4 MAs. Further, the model presented provides a powerful template to probe aza-Michael addition reactivity profiles in a variety of settings e.g., the design of targeted covalent inhibitors and polyketide biosyntheses. Both settings often utilise MAs, and may benefit from a method that directly predicts and quantifies the reactivity of different MAs towards nitrogen containing nucleophiles, without the need for TS calculations. This model allows the steric and energetic details of aza-Michael additions to be studied and elucidated using an approach that directly considers a HEI in place of a TS.

This work was supported by the Engineering and Physical Sciences Research Council (EP/L016354/1) and the University of Bath. This work was completed using the Balena HPC service at the University of Bath.

\section{Conflicts of interest}

There are no conflicts to declare.

\section{Notes and references}

1 C. Hasselgren, et al., Regul. Toxicol. Pharmacol., 2019, 107, 104403.

2 N. Gellatly and F. Sewell, Comput. Toxicol., 2019, 11, 82-89.

3 R. L. Siegel, K. D. Miller and A. Jemal, Ca-Cancer J. Clin., 2020, 70, 7-30.

4 S. J. Enoch, C. M. Ellison, T. W. Schultz and M. T. D. Cronin, Crit. Rev. Toxicol., 2011, 41, 783-802.

5 E. Eder, C. Hoffman, H. Bastian, C. Deininger and S. Scheckenbach, Environ. Health Perspect., 1990, 88, 99-106.

6 B. Ames, in Chemical Mutagens: Principles and Methods for their Detection, 1971.

7 P. Mccarren, C. Springer and L. Whitehead, Cheminf, 2011, 3, 51.

8 D. M. Sedgwick, M. N. Grayson, S. Fustero and P. Barrio, Synth., 2018, 50, 1935-1957.

9 E. H. Krenske, R. C. Petter, Z. Zhu and K. N. Houk, J. Org. Chem., 2011, 76, 5074-5081.

10 P. Townsend and M. Grayson, Chem. Res. Toxicol., DOI: 10.1021/ acs.chemrestox.0c00113.

11 M. H. Johansson, Mini Rev. Med. Chem., 2012, 12, 1330-1344.

12 A. Miyanaga, Nat. Prod. Rep., 2019, 36, 531-547.

13 P. A. Townsend and M. N. Grayson, J. Chem. Inf. Model., 2019, 59, 5099-5103.

14 T. E. H. Allen, M. N. Grayson, J. M. Goodman, S. Gutsell and P. J. Russell, J. Chem. Inf. Model., 2018, 58, 1266-1271.

15 S. J. Enoch and D. W. Roberts, Chem. Res. Toxicol., 2013, 26, 767-774. 16 N. Agmon, J. Chem. Soc., 1978, 74, 388-404.

17 B. A. Freeman, R. E. Wilson, R. G. Binder and W. F. Haddon, Mutat. Res., Genet. Toxicol. Environ. Mutagen., 2001, 490, 89-98.

18 A. Pérez-Garrido, A. M. Helguera, F. G. Rodríguez and M. N. D. S. Cordeiro, Dent. Mater., 2010, 26, 397-415.

19 OECD, OECD QSAR Toolbox, http://www.oecd.org/chemicalsafety (Acc. August 2020).

20 D. Gillingham, S. Geigle and O. Anatole Von Lilienfeld, Chem. Soc. Rev., 2016, 45, 2637-2655.

21 Macromodel, Schrödinger, New York, NY, 2019.

22 M. J. Frisch, et al., Gaussian 16 (Rev A.03), Gaussian Inc., Wallingford, CT, 2016.

23 M. N. Grayson, J. Org. Chem., 2017, 82, 4396-4401.

24 R. Rittner, L. C. Ducati, C. F. Tormena, B. C. Fiorin and C. B. Braga, Spectrochim. Acta, 2011, 79, 1071-1076.

25 S. S. Stoyanov, D. Y. Yancheva and B. A. Stamboliyska, Comput. Theor. Chem., 2014, 1046, 57-63.

26 W. Thiel, Wiley Interdiscip. Rev.: Comput. Mol. Sci., 2014, 4, 145-157.

27 J. P. McNamara and I. H. Hillier, Phys. Chem. Chem. Phys., 2007, 9, 2362-2370.

28 F. Gramatica, P. Vighi and M. Consolaro, Chemosphere, 2001, 42, 873-883.

29 R. S. Hunter and G. D. Sinks, SAR QSAR Environ. Res., 1995, 3, 27-36. 30 L. Simón and J. M. Goodman, Org. Biomol. Chem., 2011, 9, 689-700.

31 G. Patlewicz, A. O. Aptula, E. Uriarte, D. W. Roberts, P. S. Kern, G. F. Gerberick, I. Kimber, R. J. Dearman, C. A. Ryan and D. A. Basketter, SAR QSAR Environ. Res., 2007, 18, 515-541.

32 J. Kazius, R. McGuire and R. Bursi, J. Med. Chem., 2005, 48, 312-320.

33 OECD, SIDS Assessment Report - 3-Methyl-2-Butenal, Arona, Italy, 2003.

34 R. Benigni, L. Conti, R. Crebelli, A. Rodomonte and M. R. Vari, Environ. Mol. Mutagen., 2005, 46, 268-280.

35 K. R. Sumalatha and M. Sreepriya, Int. J. Pharma Bio Sci., 2015, 6, P290-P296. 